\title{
2. Coopération avec les pays en développement
}

Catherine Schümperli Younossian et Bruno Gurtner

\section{OpenEdition \\ Journals}

Édition électronique

URL : http://journals.openedition.org/aspd/600

DOI : 10.4000/aspd.600

ISSN : 1663-9669

\section{Éditeur}

Institut de hautes études internationales et du développement

\section{Édition imprimée}

Date de publication : 1 mars 2003

Pagination : 13-38

ISBN : 2-88247-049-5

ISSN : 1660-5934

\section{Référence électronique}

Catherine Schümperli Younossian et Bruno Gurtner, « 2. Coopération avec les pays en développement », Annuaire suisse de politique de développement [En ligne], 22-1 | 2003, mis en ligne le 24 mars 2010, consulté le 08 septembre 2020. URL : http://journals.openedition.org/aspd/600 ; DOI : https://doi.org/10.4000/aspd.600 


\section{COOPÉRATION AVEC LES PAYS EN DÉVELOPPEMENT*}

$S$ UR LE PLAN INTERNATIONAL, le Comité d'aide au développement (CAD) de l'OCDE a publié une série de Lignes directrices susceptibles d'améliorer les objectifs et la coordination de l'aide au développement entre agences de développement.

Au niveau suisse, l'APD de la Suisse a atteint 1637 millions de francs en 2001, soit 0,34\% du RNB. La moyenne des pays du CAD par rapport au pourcentage du RNB est de 0,4\%; la Suisse, qui se situe pourtant au septième rang des pays donateurs, est en dessous de la moyenne des pays du CAD. Le Conseil fédéral a réaffirmé sa volonté d'atteindre cet objectif d'ici à 2010.

2002 a été proclamée année internationale de la montagne par les Nations unies, opportunité saisie par la DDC pour présenter les activités qu'elle mène dans les régions de montagne depuis de nombreuses années. Le seco a présenté sa nouvelle stratégie (Stratégie 2006) pour les années à venir: trois axes majeurs sont à relever; une meilleure concentration géographique, le développement de partenariats stratégiques et la poursuite de sa politique de mobilisation de capitaux privés.

En 2001, 444 millions de francs ont été versés par la Suisse au titre de sa coopération multilatérale au développement, qui est gérée conjointement par la DDC et le seco, ce qui correspond à environ $30 \%$ de l'APD. L'adhésion de la Suisse à l'ONU (septembre 2002) lui permet de siéger dans les organes politiques de l'institution; la coopération de la Suisse avec l'ONU s'en trouve ainsi renforcée.

Lors de l'année passée sous revue, les ONG suisses ont versé environ 504 millions de francs d'aide au développement dans les pays du Sud et de l'Est. Les ONG ont récolté auprès du public 317 millions de francs, soit environ $63 \%$ des fonds qu'elles gèrent, et ont bénéficié des contributions publiques (Confédération, cantons et communes) pour un montant de 187 millions de francs (37\%).

\subsection{COMITÉ D’AIDE AU DÉVELOPPEMENT DE L'OCDE}

\section{$\square$ Les Lignes directrices pour le développement du CAD}

Sous l'impulsion du Comité d'aide au développement (CAD) de l'OCDE, les agences de coopération tentent de préciser des objectifs stratégiques communs dans leur politique de coopération. La réduction de la pauvreté apparaît comme tel. La Déclaration du millénaire (voir encadré suivant), adoptée par 189 Etats membres des Nations unies, énonce un ensemble d'objectifs quantitatifs et qualitatifs de développement destinés à mesurer les progrès accomplis en vue de «l'éradication de la pauvreté».

* Parties 2.1 à 2.5 et 2.7 à 2.9 par Catherine Schümperli Younossian, chargée de recherche à l'iuéd. Partie 2.6 par Bruno Gurtner, économiste à la Communauté de travail des œuvres d'entraide. 


\section{Objectifs de développement du millénaire}

Les objectifs de développement du millénaire (ODM) ont été adoptés par les chefs d'Etat et de gouvernement, au Sommet du millénaire en septembre 2000. Ils s'inspirent des objectifs internationaux de développement présentés par le CAD dans son rapport Le rôle de la coopération au développement à I'aube du XXI $I^{e}$ siècle. Ces objectifs sont les suivants:

口 éradiquer l'extrême pauvreté et la faim;

$\checkmark$ universaliser l'enseignement primaire;

口 promouvoir l'égalité des sexes et l'autonomie des femmes;

๑ faire baisser la mortalité infantile;

๑ améliorer la santé maternelle;

- combattre le VIH/sida, le paludisme et autres maladies;

口 garantir la viabilité écologique;

口 instaurer un partenariat mondial au service du développement.

Un système d'évaluation permet de mesurer les résultats obtenus dans la mise en œuvre de la Déclaration. Pour chacun des huit buts, une série d'indicateurs a été approuvée, permettant ainsi une évaluation quantitative. De plus, chacun des Etats ayant adopté la Déclaration doit fournir des rapports de mise en œuvre (Millenium Development Goals Country Reports).

Site Internet: $<$ www.un.org/french/milleniumgoals/>.

Pour atteindre ces objectifs, les membres du CAD ont élaboré un ensemble de Lignes directrices (présentées ci-dessous) en vue de coordonner et d'améliorer leurs efforts individuels et collectifs. La finalité de ces lignes directrices est notamment d'unifier et d'augmenter l'efficacité et la cohérence de la coopération au développement des membres du CAD. Elles ont été adoptées par les ministres en charge de la coopération lors de la réunion de haut niveau du CAD, les 25 et 26 avril 2001, à Paris (voir encadré page suivante).

Ce sont les Lignes directrices pour la réduction de la pauvreté qui revêtent le plus d'importance sur le plan politique. Elles sont le fruit d'une consultation avec la Banque mondiale, le Fonds monétaire international et le Programme des Nations unies pour le développement. Selon leurs auteurs, elles «attestent d'un consensus international naissant, d'une volonté partagée et d'une perception commune des moyens qui s'offrent à nous d'aider ensemble, de façon plus efficace, nos partenaires en développement à faire reculer la pauvreté ${ }^{1}$. Le document comporte quatre principaux chapitres. Le premier est consacré à la notion de pauvreté et à ses principales dimensions. Le second insiste sur la nécessité d'édifier des partenariats entre les agences de coopération et le pays partenaire, notamment en vue de l'élaboration de stratégies nationales de lutte contre la pauvreté. Le troisième chapitre est consacré à la présentation d'exemples concrets (Good Practices) de formulation, mise en œuvre et suivi de stratégies nationales centrées sur des objectifs de réduction de pauvreté. Enfin, le quatrième chapitre s'adresse aux pays donateurs afin de les encourager à une meilleure cohérence des politiques tant à l'intérieur de leur administration que dans leur politique étrangère.

1 OCDE, Les lignes directrices du CAD. La réduction de la pauvreté, OCDE, Paris, 2001. 


\section{Les lignes directrices du CAD}

- La réduction de la pauvreté (DAC Guidelines on Poverty Reduction), 2001.

- Renforcer les capacités commerciales au service du développement (Strengthening Trade Capacity for Development), 2001.

- Stratégies de développement durable (Strategies for Sustainable Development), 2001.

- Prévenir les conflits violents: quels moyens d'action? (DAC Guidelines: Helping Prevent Violent Conflict), 2001.

- Intégrer les conventions de Rio pour la coopération au développement (Integrating Rio-Convention into Development Co-operation), 2002, ligne directrice du CAD adoptée en vue de la conférence de Johannesburg.

Site Internet: $\langle w w w . o e c d . o r g / d a c>$.

La DDC a organisé un séminaire consacré aux Lignes directrices pour la réduction de la pauvreté du CAD2, au cours duquel Marco Rossi, chef de la section développement social, a évoqué le défi que représente la prise en compte de ces Lignes directrices dans la pratique de la DDC. Plusieurs arguments ont été évoqués, notamment la densité du document, abordant de très nombreux aspects qu'une agence de coopération telle que la DDC, avec des moyens limités, ne peut intégrer tels quels. Un nombre plus restreint de domaines d'intervention doit être choisi en fonction de son savoir-faire, de sa pratique et des moyens financiers à disposition. D'autre part, ces Lignes viennent s'ajouter à diverses stratégies déterminées précédemment de manière interne par la DDC ou le seco; dès lors, elles peuvent être perçues par certains collaborateurs comme une contrainte supplémentaire, sans qu'il y ait aucune objection quant au fond. Mais de l'avis général, ce texte garde sa pertinence comme document de référence et de synthèse d'expériences conduites en vue de la réduction de la pauvreté3.

\section{$\square$ Entrée en vigueur de la Recommandation sur le déliement de l'aide}

Depuis sa création, le CAD ne cesse de rechercher des moyens d'améliorer l'efficacité de l'aide; dans ce contexte un aspect important concerne le déliement de l'aide. On parle d'aide liée lorsque les achats de biens et de services, financés par les fonds d'aide publique au développement d'un pays donné, doivent être effectués auprès de fournisseurs nationaux. L'aide est non liée quand les commandes peuvent être soumises au niveau international et attribuées par adjudication aux fournisseurs les plus compétitifs.

C'est lors de la réunion de haut niveau d'avril 2001, et après plus de trois ans de négociations ardues, que le CAD est parvenu à émettre une recommandation visant à promouvoir le déliement de l'APD 4 aux pays les moins avancés (PMA). La recommandation ${ }^{5}$, qui a pris effet le $1^{\text {er }}$ janvier 2002, a pour objectif de délier l'aide destinée aux PMA. Elle fixe des procédures rigoureuses en matière de

2 Challenges for Switzerland Development Co-operation, séminaire organisé par la DDC, le 26 septembre 2002, Berne.

3 Entretien téléphonique avec Marco Rossi, chef de la section développement social, 8 octobre 2002.

4 Est classée dans l'APD l'aide assortie d'un élément de libéralité au moins égal à $25 \%$ consentie à des fins de développement par des organismes publics des pays donateurs.

5 Le texte de la recommandation est disponible sur le site Internet du CAD: <www.oecd.org/dac $>$, ou dans le rapport annuel du CAD, Rapport 2001, Paris: OCDE, 2002, pp. 54-57. 
transparence, d'application et d'examen afin de permettre l'instauration et le maintien de règles du jeu équitables, un suivi du degré de respect de ses dispositions et l'évaluation de son efficacité. Dorénavant, les biens et les services en rapport avec les projets bilatéraux d'aide au développement dans les pays les plus pauvres pourront donc être achetés sur le marché libre, dans le monde entier et en particulier dans les PVD, ce qui contribuera à une utilisation plus efficace des fonds d'aide au développement ${ }^{6}$.

Dans le Rapport sur la politique économique extérieure 2001 du Conseil fédéral, il est relevé que la Suisse a toujours fait partie des pays prônant la renonciation à l'aide liée et s'est conformée dans la pratique à cette optique (voir tableau 1). Et le rapport de relever que le consensus réalisé au sein du CAD ouvrira, aux entreprises suisses également, de nouvelles possibilités sur le marché en leur permettant de répondre aux appels d'offres provenant d'autres pays donateurs ${ }^{7}$.

Tableau 1: Degré de liaison de I'APD des pays du CAD, 2000, aide bilatérale (en pourcentage)

\begin{tabular}{|c|c|c|c|c|}
\hline & Déliée $^{a}$ & Partiellement déliée $^{b}$ & Liée $^{c}$ & Total \\
\hline Portugal & 98.2 & 1.0 & 0.8 & 100 \\
\hline Norvège & 97.7 & - & 2.3 & 100 \\
\hline Luxembourg & 96.7 & - & 3.3 & 100 \\
\hline Suède & 85.4 & 10.9 & 3.7 & 100 \\
\hline Pays-Bas & 95.3 & 0.6 & 4.1 & 100 \\
\hline Suisse & 93.6 & - & 6.4 & 100 \\
\hline France & 68.0 & 25.5 & 6.6 & 100 \\
\hline Allemagne & 93.2 & - & 6.8 & 100 \\
\hline Royaume-Uni & 91.5 & - & 8.5 & 100 \\
\hline Finlande & 89.5 & - & 10.5 & 100 \\
\hline Belgique $^{d}$ & 39.0 & 49.1 & 11.9 & 100 \\
\hline Japon & 86.4 & 0.5 & 13.1 & 100 \\
\hline Danemark & 80.5 & - & 19.5 & 100 \\
\hline Australie & 77.4 & - & 22.6 & 100 \\
\hline Autriche & 59.2 & - & 40.8 & 100 \\
\hline Espagne $^{\mathrm{e}}$ & 47.2 & 0.1 & 52.7 & 100 \\
\hline Italie & 38.2 & - & 61.8 & 100 \\
\hline Canada & 24.9 & - & 75.1 & 100 \\
\hline Grèce & 23.5 & - & 76.5 & 100 \\
\hline Etats-Unis & - & - & - & non notifié \\
\hline Irlande & - & - & - & non notifié \\
\hline Nouvelle-Zélande & - & - & - & non notifié \\
\hline Total du CAD & $(80.8)$ & (3.0) & (16.2) & 100 \\
\hline
\end{tabular}

Source: CAD, Rapport 2001, Paris: OCDE, 2002, tableau 23, p. 281.

Remarque: Engagements (à l'exclusion de la coopération technique et des dépenses administratives).

a Aide non liée: aide publique au développement servant à financer des achats de biens et services qui peuvent être effectués en totalité et librement dans la quasi-totalité des pays.

b Aide partiellement déliée: aide publique au développement servant à financer des achats de biens et services qui doivent être effectués soit dans le pays donneur, soit parmi un groupe restreint d'autres pays, lequel doit toutefois comprendre la totalité des pays bénéficiaires.

c Aide liée: aide publique au développement servant à financer des achats de biens et services effectués dans le pays donneur.

d Versements bruts (données de 1999, y compris la coopération technique et les dépenses administratives).

e Versements bruts.

6 Le paragraphe 7i) de la recommandation en précise le champ d'application: soutien à la balance des paiements et aide à l'ajustement structurel, remises de dette, aide-programme sectorielle et plurisectorielle, aide au titre de projet d'équipement, contrats de services commerciaux et APD consentie à des ONG pour des activités impliquant la passation de marchés.

7 Rapport sur la politique économique extérieure 2001, point 4121. 


\section{$\square$ Réunions annuelles du CAD}

Réunion à haut niveau du Comité d'aide au développement, mai 2002

Les ministres de la coopération pour le développement et les responsables des organismes d'aide se sont retrouvés à Paris les 15 et 16 mai 2002 pour la réunion annuelle du $\mathrm{CAD}$ à haut niveau. Deux thèmes majeurs étaient à l'ordre du jour: le commerce et le développement d'une part et, d'autre part, l'initiative du Nouveau partenariat pour le développement de l'Afrique (NEPAD).

Les participants à la réunion de haut niveau ont souligné les grands espoirs qu'ouvrent pour le développement le programme de Doha, adopté par la Conférence ministérielle en novembre 2001 et le Consensus de Monterrey, entériné à la Conférence internationale sur le financement du développement en mars 2002. Les membres du CAD ont également insisté sur le fait que les efforts doivent maintenant être centrés sur la mise en œuvre des engagements pris lors de ces deux conférences, tant au niveau des politiques de coopération que dans l'amélioration de la cohérence des politiques influant sur les perspectives de développement, en particulier dans les domaines des échanges, de l'agriculture, de l'environnement et du développement. A titre d'exemple, la réduction décisive des droits de douane élevés et des subventions agricoles visant des produits grâce auxquels les pays en développement les plus pauvres ont la capacité de se doter de solides secteurs d'exportation sera un souci prioritaire des pays en développement lors des négociations qui s'engageront dans le prolongement de la Conférence de Doha. De fait, les ministres des pays de l'OCDE ont renouvelé leur engagement de rejeter le recours au protectionnisme ${ }^{8}$.

DC] ASTM 2002, programme de Doha, pp. 152-159.

Ad Annuaire 2003, $\mathrm{n}^{\circ}$ 1, Consensus de Monterrey, sous-chap. 7.5.

Parmi les thèmes abordés lors de la réunion à haut niveau du CAD a figuré la situation des «pays peu performants» (appelés également poor performing countries ou «pays dont l'Etat est en faillite», ou encore «pays à faible revenu assujettis au stress »). Un consensus s'est dégagé pour dire que, malgré les risques encourus, les pays donateurs doivent rester présents. Un débat a été ouvert visant à revoir les approches de l'affectation de l'aide qui reposent uniquement sur l'efficacité en matière de réduction de la pauvreté à court terme afin de prendre en compte les contraintes inhérentes aux situations de partenariat difficile et de reconstruction au lendemain d'un conflit.

Les membres du CAD ont réservé un accueil très favorable à l'analyse et aux recommandations présentées dans le document intitulé La coopération pour le développement dans des situations de partenariat difficile, qui propose un ensemble de grands principes devant étayer l'action face à une situation de partenariat difficile. Les efforts doivent s'articuler autour des grands axes suivants:

๖ promouvoir l'instauration de conditions, et notamment le renforcement des capacités de nature à rendre les systèmes politiques mieux à même de tenir compte de l'opinion et des intérêts légitimes des pauvres;

8 OCDE, Réunion à haut niveau du Comité d'aide au développement, 15 et 16 mai 2002, communiqué de presse, Paris, 17.5.02. 
- maintenir l'aide destinée aux services profitant aux pauvres lorsqu'il existe des organismes gouvernementaux et non gouvernementaux dont le fonctionnement demeure efficace malgré des conditions défavorables;

๖ renforcer la coordination entre donateur et la cohérence des politiques, ce qui requiert un effort particulier dans des conditions aussi difficiles.

La question du partenariat difficile est un sujet d'importance pour la Suisse, qui concentre son aide sur des pays très pauvres qui connaissent, à des degrés divers, des problèmes de faiblesse institutionnelle et de mauvaise gouvernance. Ainsi la Suisse, qui fonde sa coopération sur des partenariats à long terme, ne veut pas, sous prétexte de rechercher une plus grande efficacité, réaliser des transferts de ressources à court terme vers des pays qualifiés de «bons performants ». Dès lors, la DDC participe activement aux travaux du CAD visant à développer une stratégie à long terme avec les pays dits «peu performants»?

Réunion ministérielle de l'OCDE, mai 2002

Parallèlement à la réunion annuelle du CAD s'est tenue la réunion annuelle du Conseil de l'OCDE au niveau des ministres, consacrée cette année au commerce et au développement. Lors de cette réunion, les ministres ont adopté une déclaration intitulée «Pour un programme d'action commun de l'OCDE au service du développement», qui renforce le rôle dévolu à l'OCDE en ce qui concerne la promotion de la cohérence des politiques publiques au service du développement, le soutien des capacités de gouvernance et d'élaboration des politiques des pays en développement, l'amélioration de l'efficacité de l'aide, l'adéquation de son volume, et le renforcement du partenariat et de la transparence.

Ces réunions simultanées ont accueilli des représentants du NEPAD venus débattre lors des deux réunions de l'OCDE de la forme que pourrait prendre le soutien futur de l'OCDE à cette initiative. Le NEPAD est une initiative africaine qui présente une approche globale et intégrée du développement pour le continent. Les principes fondamentaux sur lesquels repose le NEPAD sont: responsabilité, appropriation par l'Afrique, intégration régionale, facilitation de l'accès aux marchés, liberté économique, transparence, bonne gouvernance, Etat de droit, prévention et règlement des conflits, respect des droits de l'homme, investissement dans le capital humain, lutte contre la pauvreté et la faim ${ }^{10}$.

\section{$\square$ Rapport 2001 sur la coopération au développement}

Le rapport ${ }^{11}$ met en évidence la contribution apportée par le CAD à l'amélioration de l'efficacité de l'aide. Il cite notamment à cet égard l'accord sur le déliement de l'aide aux pays les moins avancés et l'adoption de quatre nouveaux ensembles de lignes directrices (voir supra). Des chapitres distincts sont aussi consacrés aux moyens qu'ont les donateurs d'aider les pays en conflit et à l'exploitation qui peut être faite de l'économie du savoir et des opportunités du numérique pour relancer le développement.

9 DAC, Development Co-operation in Difficult Partnership, doc. DCD/DAC (2002)11/REV1, May 2002.

10 OCDE, L'OCDE et le nouveau partenariat pour le développement de l'Afrique, communiqué de presse, 15-16.5.02.

11 OCDE, Les dossiers du CAD - Coopération pour le développement - Rapport 2001, volume 3, $\mathrm{n}^{\mathrm{o}}$ 1, Paris : OCDE, 2002. 


\subsection{VOLUME DE L'APD DES PAYS DU CAD ET DE LA SUISSE}

\section{$\square A P D$ fournie par l'ensemble des membres du CAD}

L'aide publique au développement (APD) des pays du CAD aux pays en développement s'est élevée, en 2001, à 51,4 milliards de dollars ${ }^{12}$. La baisse de l'APD totale de 53,7 milliards de dollars en 2000 à 51,4 milliards en 2001 résulte en partie de la chute des taux de change de certaines monnaies vis-à-vis du dollar. En termes réels, l'APD est restée relativement stable, avec une légère baisse de 1,4\%. L'APD des pays membres de l'Union européenne (UE) et des Etats-Unis augmente, compensant la baisse de l'APD du Japon.

Tableau 2: APD de l'ensemble des pays du CAD (1994-2001)

\begin{tabular}{lrrrrrrrr}
\hline APD nette du CAD & $\mathbf{1 9 9 4}$ & $\mathbf{1 9 9 5}$ & $\mathbf{1 9 9 6}$ & $\mathbf{1 9 9 7}$ & $\mathbf{1 9 9 8}$ & $\mathbf{1 9 9 9}$ & $\mathbf{2 0 0 0}$ & $\mathbf{2 0 0 1}$ \\
\hline Milliards de dollars & 59.20 & 58.90 & 55.40 & 48.30 & 51.90 & 56.40 & 53.70 & 51.40 \\
\hline $\begin{array}{l}\text { Performance moyenne des } \\
\text { pays du CAD (\% APD/RNB) }\end{array}$ & 0.45 & 0.41 & 0.39 & 0.38 & 0.39 & 0.39 & 0.39 & 0.40 \\
$\begin{array}{l}\text { APD des pays du CAD } \\
\text { en \% du RNB }\end{array}$ & 0.29 & 0.27 & 0.25 & 0.22 & 0.23 & 0.24 & 0.22 & 0.22 \\
\hline
\end{tabular}

Source: OCDE/CAD, Coopération pour le développement, Rapport annuel, communiqué de presse, mai 2002.

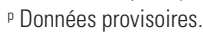

Les Etats-Unis ont augmenté leur APD à 10,9 milliards de dollars et sont devenus le premier pays donateur pour la première fois depuis 1992. Leur aide a passé de 0,10 à $0,11 \%$ du $\mathrm{RNB}^{13}$. Les donateurs les plus importants suivants sont le Japon (9,7 milliards de dollars), suivi par l'Allemagne, le Royaume-Uni, la France et les Pays-Bas, contribuant à un total de l'UE de 26 milliards de dollars. A relever que le Danemark, la Norvège, les Pays-Bas, le Luxembourg et la Suède continuent à être les seuls à atteindre l'objectif d'APD fixé par les Nations unies de $0,7 \%$ du RNB.

Treize des vingt-deux pays membres du CAD, dont neuf pays membres de l'UE, ont notifié une hausse en termes réels de leur APD. L'Espagne, l'Irlande et le Luxembourg ont connu les plus fortes croissances en termes réels. La plus grande partie de la hausse de l'aide des Etats-Unis en 2001 est due à un soutien économique de 600 millions de dollars au Pakistan suite aux événements du 11 septembre 2001. L'APD du Japon a baissé de presque 4 milliards de dollars. L'un des facteurs les plus importants dans cette chute a été la dépréciation de $12,7 \%$ du yen. Les autres facteurs étaient le calendrier des contributions aux organisations multilatérales et des remboursements de prêts de la part de pays d'Asie dont les économies ont repris depuis la crise financière asiatique. En termes réels, l'APD du Japon a diminué de $18 \%$. En comparaison internationale (selon les pays membres du CAD), la Suisse occupe le septième rang de l'APD en pourcentage du RNB $(0,34 \%)$, mais elle se situe au-dessous de la moyenne des pays du CAD dont la performance moyenne se situe à $0,4 \%$ du RNB (pour de plus amples détails, se reporter à la partie «Statistiques»).

12 OCDE, Une image contrastée de l'aide publique au développement en 2001 : les Etats-Unis redeviennent le principal pays donneur; l'aide de la plupart des pays de l'UE augmente, Paris, 13.5.02.

13 Revenu national brut (RNB): nouvelle notion de référence introduite par l'OCDE en 2001. 


\section{$\square$ APD fournie par la Suisse}

En 2001, l'APD ${ }^{14}$ de la Suisse a atteint 1531 millions de francs, soit $0,34 \%$ du RNB. L'APD comprend l'ensemble des flux financiers qui proviennent de la Confédération, des cantons et des communes destinés aux pays en développement et figurant dans la liste établie par le CAD (voir la liste à la fin de l'ouvrage). L'aide bilatérale a atteint 1087 millions et l'APD multilatérale 444 millions. Comparée à l'année 2000 (1503 millions de francs), l'APD n'a subi qu'une très légère augmentation en chiffres absolus, sans toutefois influencer le pourcentage du RNB. Elle reste encore loin de l'objectif de 0,4\% du RNB énoncé par le Conseil fédéral. L'aide publique ${ }^{15}$ de la Suisse aux pays en transition s'est élevée à 106 millions de francs.

Tableau 3: Vue d'ensemble de l'aide publique de la Suisse aux pays en développement et aux pays en transition, 2001 (en millions de francs)

\begin{tabular}{lrrrrr}
\hline & $\begin{array}{c}\mathbf{2 0 0 1} \\
\text { APD bilat. }\end{array}$ & $\begin{array}{c}\mathbf{2 0 0 1} \\
\text { APD multilat. }\end{array}$ & $\begin{array}{c}\mathbf{2 0 0 1} \\
\text { Aide publique }\end{array}$ & $\begin{array}{r}\mathbf{2 0 0 1} \\
\text { Total }\end{array}$ & $\begin{array}{r}\mathbf{2 0 0 0} \\
\text { Total }\end{array}$ \\
\hline Confédération & $\mathbf{1 0 6 5 , 8}$ & $\mathbf{4 4 3 . 8}$ & $\mathbf{1 0 4 . 3}$ & $\mathbf{1 6 1 3 , 9}$ & $\mathbf{1 5 8 6 , 8}$ \\
\hline DDC & 809.0 & 365.2 & 45.8 & 1220,1 & 1156,6 \\
\hline Aide humanitaire & 217.7 & 28.5 & 9.4 & 255.7 & 262.1 \\
\hline \multicolumn{1}{c}{ Coopération au développement } & 526.4 & 336.7 & 1.7 & 864.8 & 817.9 \\
\hline \multicolumn{1}{c}{ Coopération avec l'Est } & 64.9 & - & 34.7 & 99.6 & 76.6 \\
\hline seco & 149.2 & 25.9 & 53.0 & 228.1 & 254.9 \\
\hline \multicolumn{1}{c}{ Coopération au développement } & 93.7 & 25.9 & - & 119.5 & 129.8 \\
\hline \multicolumn{1}{c}{ Coopération avec l'Est } & 55.5 & - & 53.0 & 108.5 & 125.1 \\
\hline Autres départements & 107.6 & 52.7 & 5.4 & 165.7 & 175.3 \\
\hline Cantons et communes & $\mathbf{2 1 . 3}$ & - & $\mathbf{1 . 7}$ & $\mathbf{2 3 . 0}$ & $\mathbf{2 4 . 2}$ \\
\hline \multicolumn{1}{c}{ Aide humanitaire } & 3.6 & - & 0.2 & 3.8 & 5.2 \\
\hline \multicolumn{1}{c}{ Coopération au développement } & 17.7 & - & 1.5 & 19.2 & 19.0 \\
\hline Total & $\mathbf{1 0 8 7 , 1}$ & $\mathbf{4 4 3 , 8}$ & $\mathbf{1 0 6 . 0}$ & $\mathbf{1 6 3 6 , 9}$ & $\mathbf{1 6 1 1 , 0}$ \\
\hline Dont: Aide humanitaire & 295.2 & 28.5 & 9.6 & 333.4 & 346.7 \\
\hline Coopération au développement & 671.4 & 415.3 & 8.6 & 1095,3 & 1062,5 \\
\hline Coopération avec l'Est & 120.4 & - & 87.7 & 208.1 & 201.7 \\
\hline Aide publique au dévelop. (APD) & 1087,1 & 443.8 & & 1530,9 & 1502,7 \\
\hline
\end{tabular}

Source: Service statistique de la DDC, octobre 2002.

\subsection{COOPÉRATION AU DÉVELOPPEMENT DE LA SUISSE EN 2001}

\section{$\square$ Objectifs énoncés de la politique d'aide de la Suisse}

La politique de coopération au développement s'appuie sur quatre «piliers », qui définissent les orientations générales de l'aide ou en précisent les modes opératoires : les bases légales, les messages du Conseil fédéral, les rapports du Conseil fédéral, les orientations stratégiques des offices en charge de la coopération.

14 L'aide publique au développement (APD) est définie par l'OCDE comme l'ensemble des flux financiers qui proviennent d'organismes publics (Confédération, cantons et communes), accordés à des conditions de faveur (dons ou prêts à taux bonifiés) et qui ont pour but essentiel le développement économique et social des pays destinataires. Ils sont destinés à des pays ou territoires figurant dans la liste établie par l'OCDE. Cette liste comprend 152 pays et territoires classés sous l'appellation «en développement» dont, en Europe, tous les pays de l'ex-Yougoslavie, l'Albanie, la Moldova et Malte.

15 L'aide publique répond aux quatre mêmes critères que l'APD, mais elle est destinée aux pays dits en transition, soit 12 pays d'Europe centrale et orientale, et 23 pays en développement plus avancés. 


\section{Les bases légales}

La coopération au développement et l'aide humanitaire, ainsi que la politique de coopération avec les pays d'Europe de l'Est et de la Communauté des Etats indépendants, s'appuient sur deux bases légales.

- La Loi fédérale sur la coopération au développement et l'aide humanitaire internationale (1976) et son ordonnance d'application (1977). La loi définit les objectifs généraux de l'aide, à savoir contribuer en priorité à soutenir les efforts des pays en développement en vue d'améliorer les conditions de vie de leurs populations et permettre à ces pays d'être en mesure d'assurer leur propre développement. La loi précise que la coopération doit soutenir en priorité les efforts des pays en développement, des régions et des groupes de populations les plus défavorisés. Enfin, elle présente les différentes formes que peut revêtir la coopération, dont les principales sont la coopération technique, l'aide financière, les mesures de politique économique et l'aide humanitaire.

- La politique de soutien à la transition dans les pays d'Europe de l'Est et de la Communauté des Etats indépendants (CEI) est régie, quant à elle, par l'Arrêté fédéral concernant la coopération avec les Etats d'Europe de l'Est (1995).

\section{Les messages du Conseil fédéral}

Les messages du Conseil fédéral relatifs à la coopération au développement et à son financement contiennent un exposé des objectifs, des orientations politiques et opérationnelles des différents programmes, ainsi qu'une demande de crédits pluriannuels; sont périodiquement soumis à l'approbation des Chambres fédérales (Parlement). Depuis dix ans, le Parlement a approuvé une dizaine de crédits de programmes relatifs à la coopération internationale, totalisant environ 15’000 millions de francs.

Tableau 4: Liste des principaux crédits de programmes actuellement en vigueur

\begin{tabular}{|c|c|c|c|}
\hline Type & $\begin{array}{c}\text { Date } \\
\text { d'approbation }\end{array}$ & $\begin{array}{c}\text { Montants } \\
\text { (en mio. de francs) } \\
\end{array}$ & $\begin{array}{c}\text { Durée } \\
\text { minimale }\end{array}$ \\
\hline $\begin{array}{l}\text { Financement de mesures } \\
\text { de désendettement }\end{array}$ & 1991 & 400 & 5 ans \\
\hline $\begin{array}{l}\text { Programme et projets en faveur } \\
\text { de l'environnement global } \\
\text { dans les pays en développement }\end{array}$ & 1991 & 300 & 5 ans \\
\hline $\begin{array}{l}\text { Adhésion de la Suisse } \\
\text { aux institutions de Bretton Woods }\end{array}$ & 1992 & 4986 & - \\
\hline $\begin{array}{l}\text { Participation de la Suisse } \\
\text { à l'augmentation du capital des banques } \\
\text { régionales de développement }\end{array}$ & 1995 & 800 & 4 ans \\
\hline Aide humanitaire internationale & 1996 & 1050 & 4 ans (1997-2001) \\
\hline Mesures de politique économique et commerciale & 1996 & 960 & 4 ans (1997-2001) \\
\hline Coopération avec les pays d'Europe de l'Est et de la CEI & 1998 & 900 & 4 ans (1999-2002) \\
\hline $\begin{array}{l}\text { Coopération technique et financière } \\
\text { en faveur des pays en développement }\end{array}$ & 1998 & 4000 & 4 ans (1999-2002) \\
\hline Aide humanitaire internationale & 2002 & 1500 & 4 ans (2002-2005) \\
\hline Total & & 14896 & \\
\hline
\end{tabular}

Source: DDC, in Examen en matière de coopération pour le développement, Suisse, Dossier du CAD 2000, vol. 1, n 4, Paris: OCDE, 2001. 
En 2003, deux nouveaux messages seront soumis au Parlement, l'un présenté par la DDC concernant la coopération technique et financière en faveur des pays en développement, le second préparé par le seco concernant les mesures de politiques économique et commerciale.

\section{Les rapports de politique étrangère}

Le Conseil fédéral transmet régulièrement des rapports au Parlement sur les objectifs de sa politique étrangère. Parmi les rapports qui vont marquer les relations de la Suisse avec les pays en développement, signalons le Rapport sur les relations Nord-Sud de la Suisse dans les années 90, mieux connu sous le nom de Lignes directrices Nord-Sud, ainsi que d'autres rapports thématiques précisant le cadre dans lequel s'inscrit la politique extérieure de la Suisse. Ainsi on notera l'élargissement de la politique étrangère dans les domaines de la promotion de la paix, de la mise en œuvre de la nouvelle politique de sécurité et de la coopération au développement ${ }^{16}$. La question des droits de l'homme occupe également une place importante de la politique étrangère de la Suisse, comme en témoigne le Rapport sur la politique suisse des droits de l'homme ${ }^{17}$.

\section{Les stratégies des différents offices}

Les différents offices en charge de la coopération au développement et de l'aide humanitaire ont fourni un important travail de redéfinition de leurs cadres stratégiques pour les années à venir. Chacun des offices a produit un document qui vise à définir ses objectifs, principes et modalités de travail. Ces différentes stratégies s'inscrivent dans la perspective du développement durable et de la lutte contre la pauvreté et sont largement présentées dans l'Annuaire.

[D] ASTM 2001, Stratégie 2010 de la DDC (2000), pp. 319-320.

Dd Annuaire 2003, no 1, Stratégie 2006 du seco: voir sous-chap. 2.5; Stratégie 2005 de l'aide humanitaire: voir chap. 4 ; Stratégie de politique extérieure de la Suisse pour l'Europe du Sud-Est: voir chap. 1.

A noter également que la Suisse entend utiliser les Cadres stratégiques de lutte contre la pauvreté (CSLP), qui sont au cœur du nouveau plan antipauvreté de la communauté internationale ${ }^{18}$. Les CSLP constituent un mécanisme permettant de concentrer les politiques et les ressources pour favoriser la réduction de la pauvreté. Les mesures de lutte contre la pauvreté sont intégrées dans un cadre macroéconomique qui prend en compte tant l'épargne provenant des opérations de désendettement qu'une stimulation de la croissance économique, grâce à un processus de consultation étendue des acteurs nationaux. Un CSLP procure à un pays un cadre d'une durée de trois ans pour définir ses priorités politiques, ses programmes en matière de dépenses publiques et sa coopération au développement. Il définit les objectifs du pays en vue de réduire la pauvreté, les réformes structurelles nécessaires, les besoins en financement et l'établissement de priorités budgétaires. La Suisse entend utiliser le CSLP comme cadre à long terme de sa coopération au développement. Le seco et la DDC ont publié une brochure consacrée à cet instrument ${ }^{19}$.

[D] Annuaire 2003, n 1, sous-chap. 7.4, «Lutte contre la pauvreté ».

16 Rapport sur les dimensions humanitaires de la politique extérieure de la Suisse (1999), Rapport sur la politique de sécurité 2000 (1999), Rapport sur la politique de maîtrise des armements et de désarmement de la Suisse (2000).

17 Rapport sur la politique suisse des droits de l'homme du 16 février 2000.

18 DDC, «CSLP, un outil risqué mais prometteur», Newsletter, no 3, juin 2002.

19 DDC/seco, Coup d'œil aux Cadres stratégiques de lutte contre la pauvreté, 2002. A commander <sbwi@deza.admin.ch>. 


\section{Les offices fédéraux en charge de la coopération au développement}

Au niveau fédéral, deux offices sont responsables, aux termes de la loi de 1976 relative à la coopération, de la conception et de la mise en ouvre de l'aide au développement: la Direction du développement et de la coopération (DDC), qui fait partie du Département fédéral des affaires étrangères (DFAE), et le Secrétariat d'Etat à l'économie (seco), qui appartient au Département fédéral de l'économie. La DDC est chargée de la coopération technique et de l'aide financière, de l'aide humanitaire et des engagements en cas de catastrophe ainsi que, depuis 1995, de la coopération technique avec les pays d'Europe de l'Est et les Etats de la CEI. Le seco élabore et met en œuvre les mesures de politique économique et commerciale dans le cadre de la coopération au développement ainsi que de la coopération financière avec l'Europe de l'Est et les pays de la CEI. Les deux offices fédéraux assument en commun la responsabilité de l'aide financière multilatérale, mais la coordination globale du secteur incombe à la DDC. La DDC gère environ $80 \%$ des versements de l'APD et compte plus de 350 collaborateurs en Suisse et expatriés à l'étranger; quant au seco, il assure environ 15\% des versements et s'appuie sur le travail d'une cinquantaine de collaborateurs. Lors de ses évaluations, le Comité d'aide au développement (CAD) relève régulièrement les problèmes de coordination qui peuvent apparaître du fait d'une responsabilité partagée entre deux départements. Cependant, lors de sa dernière évaluation, le CAD a noté les efforts entrepris pour renforcer la coordination entre les principaux offices responsables de la coopération ${ }^{20}$.

Il faut ajouter à ces deux offices l'Office fédéral de l'environnement, des forêts et du paysage (OFEFP), qui fait partie du Département fédéral de l'environnement, des transports, de l'énergie et de la communication (DETEC). Depuis la Conférence de Rio sur l'environnement et le développement (1992), la politique environnementale internationale a pris un élan très important. De plus, la protection de l'environnement apparaît comme l'un des quatre objectifs stratégiques des relations Nord-Sud de la Suisse. C'est à l'OFEFP que reviennent la responsabilité des questions internationales d'environnement, ainsi que la représentation de la Suisse auprès des instances internationales correspondantes. Il dispose de moyens financiers relatifs à l'engagement de la Suisse dans ce domaine.

An Annuaire 2003, $\mathrm{n}^{\circ}$ 1, chap. 10.

\section{$\square 2002$ proclamée Année internationale de la montagne par l'ONU}

L'importance des régions de montagne est reconnue sur le plan international depuis le Sommet de la Terre lors duquel le développement durable de ces régions s'est vu accorder un chapitre spécial dans l'Agenda 21. Pour concrétiser cette préoccupation, l'Assemblée générale des Nations unies a décidé, en automne 1998, de proclamer l'année 2002 «Année internationale de la montagne $»^{21}$. L'Office fédéral du développement territorial (ARE) et la DDC sont les chefs de file de la campagne menée en Suisse.

La Suisse, pays de montagnes, a saisi cette occasion pour réaffirmer son engagement envers cette thématique. Depuis de nombreuses années, la Suisse s'est

$20 \mathrm{CAD}$, Examen en matière de coopération pour le développement, Suisse, Les Dossiers du CAD - Coopération pour le développement, CAD 2000, vol. 1. n 4, Paris: OCDE, 2001, p. II, 94.

21 Site officiel de l'Année internationale de la montagne : <www.mountains2002.org >. 
fortement engagée dans la mise en œuvre du chapitre de l'Agenda 21 sur les montagnes, car elle bénéficie d'une expérience de longue date dans le développement durable de ces régions. Elle a joué un rôle très actif en affrontant les questions de mise en valeur durable des montagnes à l'échelle mondiale, de même que dans la mise en place d'importants programmes de coopération au développement dans une vingtaine de pays.

Dans le cadre de l'Année internationale, la Suisse a contribué activement à la mise en place d'une série d'actions qui ont débuté en 2002 et seront développées dans les années à venir. Par cet engagement, elle entend exprimer sa solidarité avec les populations les plus pauvres de la terre, qui, dans des conditions naturelles difficiles, luttent pour leur survie au milieu d'un environnement ingrat. Parmi les manifestions d'importance, citons la Conférence internationale sur l'agriculture durable et le développement rural dans les régions de montagne $^{22}$, qui s'est tenue à Adelboden en juin 2002. Cette conférence, organisée sous les auspices de la FAO, de l'Office de l'agriculture, de la DDC et de l'ARE, a réuni plus de 200 participants du monde entier pour un échange d'informations sur les défis, les préoccupations et les expériences en la matière. Une déclaration a été adoptée à l'issue de la conférence, stipulant notamment que la mondialisation a souvent des conséquences négatives sur les régions de montagne, régions qui ont besoin d'une attention toute particulière notamment en raison de leur isolement géographique et des écosystèmes vulnérables rendant difficile la production économique. Les résultats de la conférence ont été mis à disposition du Sommet mondial du développement durable de Johannesburg en septembre 2002.

A l'occasion de l'Année internationale de la montagne, la DDC a décidé d'examiner les activités qu'elle a menées et mène encore dans des pays ou régions de montagne, ainsi qu'à un niveau global. Au cours des quarante dernières années, la DDC s'est engagée dans toute une série d'activités centrées sur le développement durable des régions de montagne dans des pays tels que le Népal, la Bolivie, le Bhoutan et plus récemment en Asie centrale. Se fondant sur ses expériences de terrain, la DDC a soutenu plus tard la création et le fonctionnement d'institutions régionales et internationales, ainsi que de réseaux permettant de réunir des compétences dans le domaine spécifique du développement durable des régions de montagne. Ces expériences sont présentées dans une publication Les Montagnes et leurs habitants parue en $2002^{23}$.

\subsection{DDC: COOPÉRATION BILATÉRALE AU DÉVELOPPEMENT EN 2001}

En 2001, la DDC a versé environ 591,3 millions de francs d'APD au titre de sa coopération bilatérale au développement. Par rapport à 2000, c'est 71,6 millions supplémentaires dépensés en faveur des pays en développement (plus 12\%). Cette augmentation s'explique par une légère augmentation régulière dans tous les pays, ainsi que par l'attribution d'un crédit supplémentaire de 10 millions de francs pour l'ex-Yougoslavie et d'une forte augmentation de l'aide au Mozambique.

22 Pour de plus amples informations sur la conférence et un accès aux principaux documents: <www.sard-m2002.ch>.

23 DDC, Les montagnes et leurs habitants, DDC, Berne, 2002. 
$\square$ Répartition géographique de l'aide, 2001

Tableau 5: Répartition géographique de l'APD bilatérale de la DDC au titre de la coopération au développement en 2001 (en millions de francs)

\begin{tabular}{|c|c|c|c|}
\hline Afrique & 159.0 & Amérique latine (suite) & \\
\hline Pays à faible revenu & 126.9 & Colombie & 2.7 \\
\hline Mozambique & 29.5 & El Salvador & 3.4 \\
\hline Tanzanie & 19.0 & Autres pays & 8.9 \\
\hline Burkina Faso & 13.0 & Non ventilé et projets régionaux & 14.5 \\
\hline Niger & 11.7 & Asie & 166.8 \\
\hline Tchad & 11.8 & Pays à faible revenu & 137.5 \\
\hline Bénin & 9.3 & Inde & 29.5 \\
\hline Mali & 7.9 & Népal & 20.9 \\
\hline Soudan & 0.3 & Bangladesh & 20.2 \\
\hline Congo (R.D.) & 0.4 & Vietnam & 14.0 \\
\hline Angola & 0.2 & Pakistan & 14.9 \\
\hline Madagascar & 5.9 & Afghanistan & 1.2 \\
\hline Rwanda & 4.7 & Tadjikistan & 4.6 \\
\hline Sénégal & 4.4 & Kirghizistan & 7.9 \\
\hline Ethiopie & 1.4 & Corée du Nord & 2.4 \\
\hline Erythrée & 0 & Chine & 2.8 \\
\hline Cameroun & 2.0 & Bhoutan & 6.4 \\
\hline Zambie & 0.1 & Indonésie & 2.5 \\
\hline Autres pays & 5.3 & Cambodge & 4.8 \\
\hline Pays à revenu intermédiaire & 10.7 & Autres pays & 5.4 \\
\hline Afrique du Sud & 8.5 & Pays à revenu intermédiaire & 14.5 \\
\hline Egypte & 0.8 & Palestine & 8.3 \\
\hline Autres pays & 1.4 & Géorgie & 1.1 \\
\hline Non ventilé et projets régionaux & 21.4 & Jordanie & 0.3 \\
\hline Amérique latine & 79.9 & Philippines & 2.1 \\
\hline Pays à faible revenu & 12.0 & Autres pays & 2.7 \\
\hline Nicaragua & 7.0 & Non ventilé et projets régionaux & 14.8 \\
\hline Haïti & 3.2 & Europe & 49.4 \\
\hline Honduras & 1.8 & Pays à revenu intermédiaire & 49.4 \\
\hline Pays à revenu intermédiaire & 53.4 & R.F. Yougoslavie (Serbie, y.c. Kosovo; Monténégro) & 12.6 \\
\hline Bolivie & 15.8 & Bosnie-Herzégovine & 10.3 \\
\hline Pérou & 11.9 & Albanie & 6.0 \\
\hline Equateur & 10.7 & Autres pays & 20.5 \\
\hline Pays à faible revenu & & & 276.4 \\
\hline Pays à revenu intermédiaire & & & 128.0 \\
\hline Non ventilé et projets régionaux & & & 50.7 \\
\hline Non ventilé par continent & & & 136.2 \\
\hline APD bilatérale, total & & & 591.3 \\
\hline
\end{tabular}

Sources: DDC/seco, Rapport annuel 2001, Berne, 2002. Service statistique de la DDC, novembre 2002

L'APD bilatérale de la DDC au titre de la coopération au développement bénéficie en grande partie à l'Afrique et à l'Asie (environ $26 \%$ dans les deux cas), puis à l'Amérique latine $(11 \%)$ et à l'Europe $(8 \%)$, le reste bénéficiant à des pays en développement sans spécification (29\%). A noter également que $47 \%$ de 1 'APD bilatérale sont attribués à des pays à faible revenu et $22 \%$ bénéficient aux pays à revenu intermédiaire, les $31 \%$ restants ne sont pas ventilés. 
2002 aura été une année de conflits dans de nombreuses régions du monde, mais en particulier dans trois pays où la coopération suisse déploie ses activités (Inde-Pakistan, Népal et Territoires palestiniens autonomes et occupés). Les relations tendues entre l'Inde et le Pakistan sur la question du Cachemire n'ont pas eu d'impact direct sur les programmes de la coopération suisse, mais les bureaux de coordination de la DDC à Islamabad et Delhi ont travaillé dans le cadre d'un dispositif de sécurité accru ${ }^{24}$. Même situation tendue au Népal, où un conflit oppose maoïstes et forces gouvernementales. La Suisse est engagée depuis plus de quarante ans dans le pays, elle finance quelque 25 projets pour environ 19 millions de francs par an. La situation politique et économique ainsi que les conditions-cadres pour la coopération au développement se sont considérablement détériorées depuis l'instauration de l'état d'urgence (novembre 2001). La DDC analyse les influences du contexte sur son programme ainsi que les adaptations nécessaires ${ }^{25}$. Enfin, les combats prévalant dans les Territoires palestiniens autonomes et occupés (TPAO) ont causé des dégâts matériels importants, chiffrés par les organisations d'aide et les experts des principaux pays donateurs à environ 600 millions de francs. Les projets financés par la Suisse avaient relativement peu souffert (situation en juin 2002). La DDC a décidé de poursuivre sa coopération au développement (formation de professionnels et éducation, développement social, droits de l'homme, bonne gestion des affaires publiques, etc.) en se concentrant davantage sur l'aide humanitaire et de survie destinée à la population directement touchée ${ }^{26}$.

\section{$\square$ Pays prioritaires de la DDC}

Depuis de nombreuses années, la DDC a adopté le principe de concentration géographique. Le choix des pays dits prioritaires s'établit en fonction de critères concernant les pays partenaires (indice de pauvreté, critère de bonne gouvernance, stabilité permettant un engagement à long terme), ainsi que du point de vue de la coopération suisse (dialogue régulier, programme à moyen terme, dialogue sur le plan politique dans les secteurs concernés, volume financier minimum). Une réorientation de la politique de concentration s'impose lorsque le contexte politique, économique et social change. Généralement, lorsqu'un pays est considéré comme prioritaire, la DDC ouvre un bureau dit de coordination (BUCO) dans le pays en question. Une des caractéristiques de la coopération bilatérale de la DDC est la constance et la durée dans ses engagements dans les pays prioritaires, comme en témoigne l'encadré page suivante, qui indique le début des activités suisses (par le biais d'une œuvre d'entraide suisse soutenue par la Confédération ou par la coopération suisse), ainsi que l'année correspondant à la décision de reconnaissance de ces pays comme «prioritaires».

24 DDC, «Tension entre l'Inde et le Pakistan, la coopération se poursuit malgré tout », Newsletter, $\mathrm{n}^{\circ} 1$, février 2002.

25 DDC, «Gestion des risques au Népal, mesures de sécurité indispensables», Newsletter, ${ }^{\circ}$ 2, avril 2002.

26 DDC, «Après la réoccupation des territoires palestiniens, la DDC se voit contrainte de renforcer son aide humanitaire », Newsletter, no 3, juin 2002. 
Pays prioritaires (Sud) de la DDC, situation en 2002

\begin{tabular}{|c|c|c|}
\hline Pays & $\begin{array}{l}\text { Début des activités suisses } \\
\text { (ONG ou Confédération) }\end{array}$ & $\begin{array}{c}\text { Décision de reconnaître } \\
\text { les pays comme «prioritaires » }\end{array}$ \\
\hline 1. Bénin & 1961 (Dahomey) & 1983 \\
\hline 2. Burkina Faso & 1974 (Haute-Volta) & 1974 \\
\hline 3. Mali & Vers 1971 & 1977 \\
\hline 4. Niger & Fin des années 60 & 1977 \\
\hline 5. Tchad & Vers 1965 & $\begin{array}{l}\text { 1983. Guerre civile 1979-1982, } \\
\text { mais les projets ont continué }\end{array}$ \\
\hline 6. Mozambique & 1979 & 1982 \\
\hline 7. Tanzanie & Milieu des années70 & 1981 \\
\hline 8. Bolivie & 1969 & 1970. Interrompu 1980-1982 \\
\hline 9. Equateur & 1969 & 1970-1978, puis à nouveau depuis1998 \\
\hline 10. Pérou & 1964 & 1964 \\
\hline 11. Nicaragua/Amérique centrale & $\begin{array}{l}\text { Honduras: } 1979 \\
\text { Nicaragua: } 1979 \\
\text { El Salvador: } 1982\end{array}$ & $\begin{array}{c}\text { Honduras: } 1981 \\
\text { Nicaragua: } 1982 \\
\text { El Salvador: } 1999\end{array}$ \\
\hline 12. Bangladesh & 1971 & 1981 \\
\hline 13. Bhoutan & 1969 & 1983 \\
\hline 14. Inde & 1961 & 1961 \\
\hline 15. Népal & 1956 & 1962 \\
\hline 16. Pakistan & 1966 & 1977 \\
\hline $\begin{array}{l}\text { 17. Région Mékong } \\
\text { (Vietnam, Laos, Thaïlande) }\end{array}$ & $\begin{array}{l}\text { Mékong: } 1968 \\
\text { Vietnam: } 1993\end{array}$ & $\begin{array}{l}\text { Mékong: } 1994 \\
\text { Vietnam: } 1997\end{array}$ \\
\hline
\end{tabular}

Sources: Site Internet de la DDC. DDC, division coopération bilatérale, sections géographiques. Rolf Wilhelm (anciennement DDC). Liste mise à jour en automne 2002.

La DDC procède à une évaluation de la situation politique, économique et sociale des pays prioritaires et, selon les résultats de son évaluation, se désengage petit à petit en supprimant la référence "pays prioritaire», lorsqu'elle considère que le pays est arrivé à un stade de développement déterminé. Tel est le cas de pays comme la Tunisie (1962-1980), le Cameroun (1964-1985) et le Kenya (19701996). Durant la période 1995-1998, la DDC a supprimé les bureaux de coordination d'Indonésie, de Thaïlande et du Cap-Vert. Mais durant cette période, elle a ouvert (1997) un bureau de coordination au Vietnam ${ }^{27}$. A noter également le cas de l'Equateur, considéré comme pays prioritaire de 1970 à 1978; à cette date la coopération suisse a estimé que les recettes de la production de pétrole suffiraient à contribuer au développement économique. Toutefois, l'Equateur a été à nouveau considéré comme pays prioritaire dès 1998. Deux cas particuliers doivent être relevés: le Rwanda (1964-1994) et Madagascar (1970-1998) ont été des pays prioritaires pour la DDC. Les événements tragiques du Rwanda et une situation politique difficile à Madagascar, à laquelle s'est ajouté l'assassinat d'un coopérant suisse, ont poussé la DDC à revoir la nature de ses relations avec ces deux pays.

Selon les circonstances, les conditions nécessaires à la mise en œuvre de programmes à long terme ne sont pas toujours réunies ou ne le sont que partiellement. Afin de pouvoir agir dans ces cas-là si le besoin s'en fait sentir ou dans le but de répondre à des priorités de politique étrangère, la DDC s'est donné les moyens de le faire en créant des programmes spéciaux. Ceux-ci ne doivent pas

27 Message du Conseil fédéral concernant la continuation de la coopération technique et de l'aide financière en faveur des pays en développement du 7 décembre 1998. 
obligatoirement satisfaire à tous les critères applicables pour les pays prioritaires et sont prévus pour une durée plus courte ${ }^{28}$. Quatre programmes spéciaux sont actuellement en cours; en Cisjordanie et dans la bande de Gaza (depuis 1993), en Afrique du Sud (depuis 1994), au Rwanda et à Madagascar.

Depuis le début des années 1990, la DDC a décidé de compléter l'approche concentration par pays par une approche régionale afin d'optimiser les synergies et de mieux tenir compte de réalités semblables dans des zones économiquement, socialement et culturellement assez homogènes. Deux programmes régionaux de concentration sont actuellement en cours : Nicaragua/Amérique centrale et Vietnam/Région du Mékong. Cette approche régionale peut prendre différentes formes comme l'extension transfrontalière de projets ou programmes ou la mise en œuvre de projets semblables dans des régions homogènes, etc. L'approche régionale n'est pas axée en premier lieu sur le soutien d'organisations régionales (qui font par ailleurs l'objet d'un soutien financier de la coopération) mais sur la mise en valeur d'expériences locales dans des régions homogènes.

Cependant, l'efficacité de la politique de concentration de la DDC est tempérée par l'évaluation du CAD. Comme le relèvent les experts, le nombre de pays prioritaires est élevé en regard de la taille du programme d'aide bilatérale de la Suisse. Cette situation est encore accentuée par le fait que le seco a ses propres priorités et intervient dans pas moins de 36 pays, dont 10 pays de concentration de la DDC seulement. Et de regretter que «tout ceci conduit à un saupoudrage de l'aide». Dans ces recommandations, le CAD encourage fortement la Suisse à maintenir ses priorités en faveur des plus pauvres, mais en revoyant la liste des pays concernés pour éviter une trop grande dispersion de l'aide et en allouant une fraction accrue de l'aide aux secteurs sociaux de base ${ }^{29}$.

\subsection{MESURES DE POLITIQUE ÉCONOMIQUE ET COMMERCIALE DU SECO EN 2001}

Le centre de prestation «Développement et transition» du Secrétariat d'Etat à l'économie ( $\mathrm{seco}$ ) est chargé de concevoir et de mettre en œuvre la coopération avec les pays en développement ou en transition. Il comprend 55 postes et dispose d'un budget annuel d'environ 250 millions de francs. Le centre de prestation est responsable, pour $15 \%$, de l'aide publique au développement et de $50 \%$ de l'assistance financière aux pays en transition. Par ailleurs, il assume la responsabilité conjointe, avec la DDC, de l'aide financière multilatérale, qui s'est montée à quelque 444 millions de francs en 2001.

\section{$\square$ Les objectifs et instruments du seco}

Le but principal du seco est la lutte contre la pauvreté, fixé dans la loi de développement de 1976. Pour atteindre ce but, le seco s'investit dans cinq domaines d'intervention prioritaires: promotion des investissements; renforcement des conditions-cadres; coopération liée au commerce; développement des infrastructures de base; renforcement de la coopération avec les banques multilatérales de développement.

28 DDC/seco, Mémorandum de la Suisse au CAD, Berne, avril 2000, p. 56.

$29 \mathrm{CAD}$, Examen en matière de coopération pour le développement, Suisse, Les Dossiers du CAD - Coopération pour le développement, CAD 2000, vol.1. n 4, OCDE, Paris, 2001, p. II.32-34. 
En 2001, le seco a versé plus de 193 millions de francs pour mettre en œuvre les mesures bilatérales de politique économique et commerciale. Le tableau ci-dessous présente les versements bilatéraux du seco pour les pays en développement (aide publique au développement - APD) et pour les pays en transition (aide publique - AP). Les engagements multilatéraux du seco se montent à 34,8 millions de francs pour 2001 (participation à la reconstitution de la BERD, 9 millions; contributions aux FMI et PTTE, 23,5 millions; ainsi qu'à divers organismes multilatéraux, 2,3 millions de francs.

Tableau 6: Versements nets d'APD/AP bilatérale du seco en 2001 (en millions de francs)

\begin{tabular}{|c|c|c|c|c|c|c|}
\hline Instruments & Afrique & Amérique latine & Asie & Europe & Non spécifié & Total \\
\hline Aide macroéconomique & 23.32 & 0.06 & 0.43 & 10.00 & 2.40 & 36.20 \\
\hline Aide à la balance des paiements & 23.32 & 0.06 & 0.43 & 10.00 & 0.51 & 34.31 \\
\hline $\begin{array}{l}\text { Désendettement } \\
\text { (assistance technique incluse) }\end{array}$ & & & & & 1.89 & 1.89 \\
\hline Financement des infrastructures & 4.98 & 0.04 & 8.75 & 48.31 & 0.25 & 62.33 \\
\hline $\begin{array}{l}\text { Financements mixtes } \\
\text { (assistance technique incluse) }\end{array}$ & 4.98 & 0.04 & 1.79 & 0.00 & 0.00 & 6.81 \\
\hline Aide financière & & & 6.96 & 48.31 & 0.25 & 55.52 \\
\hline Promotions des investissements & 10.20 & 4.55 & 9.05 & 13.9 & 19.51 & 57.21 \\
\hline Promotion des investissements & 10.20 & 4.55 & 9.05 & 12.16 & 19.51 & 55.48 \\
\hline Aide financière remboursable & & & & 1.73 & & 1.73 \\
\hline Promotion du commerce & 0.67 & 1.52 & 2.93 & 5.47 & 15.96 & 26.55 \\
\hline Frais de gestion & 0.04 & 0.00 & 0.02 & 0.20 & 13.89 & 14.16 \\
\hline Divers $^{a}$ & 0.00 & 0.00 & -0.038 & -3.49 & 0.25 & -3.28 \\
\hline Total & 39.22 & 6.17 & 21.14 & 74.38 & 52.27 & 193.17 \\
\hline
\end{tabular}

Source: Service statistique de la DDC, novembre 2002.

a $Y$ compris remboursement d'anciens prêts.

\section{$\square$ Réorientation des mesures économiques et commerciales}

Comme le montre le tableau suivant, les instruments traditionnels du seco comportent: les mesures de désendettement (24\%), l'assistance à la balance des paiements $(15,7 \%)$, les financements mixtes $(12.9 \%)$ et la promotion commerciale $(9,6 \%)$. A relever le haut pourcentage $(31,8 \%)$ pour les mesures destinées à favoriser l'engagement de ressources du secteur privé.

Tableau 7: Mesures de politique économique et commerciale du seco (1997-2001)

\begin{tabular}{lcr}
\hline Instruments du seco & en millions de francs & en \% \\
\hline Mesures destinées à favoriser & 204.2 & 31.8 \\
l'engagement de ressources du secteur privé & 154.0 & 24.0 \\
\hline Mesures de désendettement & 100.6 & 15.7 \\
\hline Aide à la balance des paiements & 83.0 & 12.9 \\
\hline Financements mixtes & 61.5 & 9.6 \\
\hline Promotion commerciale & 16.7 & 2.6 \\
\hline Produits de base & 21.7 & 3.4 \\
\hline Etudes et divers & $\mathbf{6 4 1 . 7}$ & $\mathbf{1 0 0}$ \\
\hline Total &
\end{tabular}

Source:DDC/seco, Rapports annue/s, 1997, 1998, 1999, 2000, 2001, Berne. 
En 1996, le Conseil fédéral et le Parlement ont réorienté les mesures de politique économique et commerciale ${ }^{30}$, dans le cadre de l'aide au développement en proposant notamment une série de nouveaux instruments de coopération destinés à favoriser l'engagement de ressources du secteur privé. Ces instruments ont pour but d'encourager les investissements dans les pays en développement, ainsi que le transfert de technologie. Le seco a ainsi développé une vingtaine d'instruments visant à promouvoir les investissements suisses ou étrangers vers les PVD, dont les trois principaux sont la Swiss Organization for Faciliting Investments (SOFI), la Swiss Development Finance Corporation (SDFC) et les Clean Production Centers.

Pour évaluer l'impact de ces nouveaux instruments, des évaluations externes de la SOFI et du Swiss Import Promotion Programme (SIPPO) ainsi que d'autres projets et programmes ont été réalisées. Selon le seco, ces évaluations ont donné une majorité de résultats positifs ${ }^{31}$. Les résultats des évaluations servent, entre autres, de base à l'élaboration du nouveau Message sur les mesures économiques et commerciales qui sera soumis au Parlement début 2003. L'introduction de ces nouveaux instruments avait fait l'objet de critiques des organisations de développement, qui regrettaient qu'une partie des fonds de développement soit utilisée pour aider des exportateurs suisses et des investisseurs plutôt que pour soutenir le développement durable des pays les plus désavantagés. La position des ONG a évolué depuis; admettant l'existence de ces instruments, elles réclament que soient élaborés des critères de développement et des indicateurs d'impact sur l'environnement ${ }^{32}$.

DA ASTM 2001, dossier «Promotion du secteur privé dans le cadre de la coopération au développement».

\section{$\square$ seco - Stratégie 2006}

En mars 2002, le seco a présenté sa nouvelle stratégie ${ }^{33}$ pour les quatre années à venir dans le domaine du développement et de la transition. La Stratégie 2006 vise à clarifier et renforcer le cadre d'analyse et de référence de la contribution du seco à la politique suisse de coopération internationale. L'objectif énoncé est la réduction de la pauvreté dans le contexte d'une économie mondiale dynamique. Pour atteindre cet objectif, le seco entend promouvoir l'économie de marché, favoriser une croissance soutenue et durable dans les pays partenaires et soutenir les réformes favorisant une intégration accrue des pays en développement ou en transition dans l'économie mondiale. Lors de la phase préparatoire, le document a fait l'objet d'une large consultation au sein de l'administration fédérale, auprès de la Commission consultative pour le développement et la coopération internationale (commission Ming), ainsi qu'auprès des milieux économiques et des ONG.

La Stratégie 2006 prévoit avant tout de se focaliser sur une meilleure concentration géographique et sur le développement de partenariats stratégiques, ainsi

30 Message du Conseil fédéral concernant la continuation du financement et la réorientation des mesures de politique économique et commerciale au titre de la coopération au développement du 29 mai 1996.

31 Knapp Oscar, «Nouvelle stratégie du seco dans le domaine du développement et de la transition », $L a$ Vie économique, mai 2002.

32 Annuaire Suisse-Tiers Monde 2001, dossier «Promotion du secteur privé dans le cadre de la coopération au développement», p. 31.

33 seco, Domaine de prestation Développement et transition - Stratégie 2006, Berne, mars 2002. 
que de continuer à mobiliser des capitaux privés. Selon le document, le seco va réduire, d'ici à 2006, le nombre de pays d'intervention, une cinquantaine actuellement, et se concentrer sur un total de 26 pays. Ces pays devront absorber $80 \%$ des dépenses et $90 \%$ des ressources humaines qui sont engagées chaque année sur des opérations bilatérales par pays. Concernant les partenariats, le document insiste sur l'importance d'une coordination internationale afin d'améliorer la qualité et l'efficacité de l'aide. Et de souligner l'importance d'un «dialogue plus intensif entre le seco et ses partenaires suisses et internationaux pour renforcer la cohérence de la politique envers les pays en développement et en transition. Au travers de mécanismes de consultation et de coordination, il s'agit de promouvoir autant que faire se peut une plus grande harmonisation entre la politique de coopération au développement et les politiques dans les domaines du commerce, de l'environnement, de l'agriculture, du marché du travail et de la migration». Parmi ses principes opérationnels, la Stratégie 2006 relève l'importance de renforcer les synergies et les processus de consultation avec les autres domaines de prestation du seco. De plus, elle souligne la nécessité de renforcer la consultation, la coordination et les synergies avec d'autres offices de l'administration fédérale (DDC, AFF, OFEFP, Direction politique/DFAE, Office fédéral de l'agriculture, etc.).

\subsection{COOPÉRATION MULTILATÉRALE}

La coopération internationale de la Suisse a toujours été basée sur une double approche, bilatérale et multilatérale. Il s'agit de deux volets d'une seule et même politique. Tandis que la coopération bilatérale garde toute son importance dans l'appui à des projets individuels au niveau des pays, la coopération multilatérale s'impose pour s'attaquer à des problèmes plus complexes qui ont souvent des ramifications aux niveaux régional et global ${ }^{34}$.

La Suisse consacre actuellement environ $70 \%$ de son aide publique au développement (APD) à la coopération bilatérale; environ $30 \%$ vont (sous forme de contributions générales) à des institutions et des programmes multilatéraux. En 2001, la coopération multilatérale de la Suisse s'est élevée à 444 millions de francs; elle est du ressort de la DDC et/ou du seco, selon les organisations concernées. Par rapport à 2000, cette forme de coopération est en légère augmentation (24 millions de francs, soit plus $5 \%$ ).

Dans l'allocation de ses ressources multilatérales, la Suisse prend en compte des critères tels que le rôle et les politiques des institutions qu'elle appuie, la complémentarité de leurs activités avec les programmes bilatéraux de la Suisse, les possibilités pour la Suisse de participer à la définition des politiques et des stratégies et à la supervision des activités de ces institutions; la qualité de leurs opérations sur le terrain; la répartition du fardeau financier entre donneurs.

L'adhésion à l'Organisation des Nations unies permet à la Suisse de siéger dans les organes politiques de l'institution et de participer à des débats et des prises de décision qui peuvent avoir d'importantes implications, entre autres sur l'ensemble des activités opérationnelles du système onusien. Mais la Suisse va aussi pouvoir

34 Chapatte Serge, in «La division de la politique de développement et coopération multilatérale: une des principales voix de la DDC», La Vie économique, seco, novembre 2001. 
tirer profit des activités de l'ONU pour ses propres programmes et projets. Une brochure de la DDC présente les différentes facettes de cette coopération ${ }^{35}$.

$\square$ Contributions multilatérales de la Suisse au titre de l'aide publique au développement

En 2001, la Suisse a soutenu les institutions suivantes:

- les fonds et programmes ainsi que certaines agences spécialisées des Nations unies, dont le PNUD, l'UNICEF, le FNUAP, l'OMS, etc. (avec des contributions totalisant 140 millions de francs);

- le Groupe de la Banque mondiale, les banques régionales de développement et le Fonds international du développement (avec des contributions totalisant 267 millions de francs);

- d'autres institutions, dont le CGIAR, l'UICN, le FEM/GEF et l'AIF (avec des contributions d'un total de 37 millions de francs).

Tableau 8: Participation de la Suisse à la coopération au développement et à l'aide humanitaire multilatérales 2001 (en millions de francs)

\begin{tabular}{lr}
\hline Institutions des Nations unies & 139.9 \\
\hline Programme des NU pour le développement (PNUD) & 52.0 \\
\hline Fonds des NU pour l'enfance (UNICEF) & 17.0 \\
\hline Haut-commissariat pour les réfugiés (HCR) & 13.0 \\
\hline Fonds des NU pour les activités en matière de population (FNUAP) & 12.0 \\
\hline Office de secours et de travaux pour les réfugiés de Palestine (UNRWA) & 11.1 \\
\hline Programmes spéciaux de I'Organisation mondiale de la santé (OMS) & 3.6 \\
\hline Programme commun des NU sur le VIH/sida (ONUSIDA) & 4.0 \\
\hline Fonds des NU pour les femmes (UNIFEM) & 0.8 \\
\hline Programme de volontaires des NU (VNU) & 0.6 \\
\hline Autres organisations des NU & 25.8 \\
\hline Groupe de la Banque mondiale, banques régionales et FIDA & 267.4 \\
\hline Banque africaine de développement (BAfD), participation au capital & 1.9 \\
\hline Fonds africain de développement (FAfD) & 46.0 \\
\hline Banque asiatique de développement (BAsD), participation au capital & 0.6 \\
\hline Fonds asiatique de développement (FAsD) & 13.9 \\
\hline Banque interaméricaine de développement (BID), participation au capital & 1.6 \\
\hline Fonds en faveur des pays pauvres très endettés (PPTE) & 23.5 \\
\hline Association internationale de développement (AID/IDA) & 140.0 \\
\hline Fonds international de développement agricole (FIDA) & 17.7 \\
\hline Autres institutions financières de développement & 22.2 \\
\hline Autres institutions multilatérales & 36.5 \\
\hline Groupe consultatif pour la recherche agricole internationale (CGIAR) & 11.1 \\
\hline Fonds pour l'environnement mondial (FEM) & 10.6 \\
\hline Global Fund to Fight AIDS, Tuberculosis and Malaria (GF-ATM) & 5.0 \\
\hline Agence intergouvernementale de la francophonie (AIF) & 4.4 \\
\hline Union internationale pour la conservation de la nature (UICN) & 1.2 \\
\hline Autres institutions multilatérales & 4.2 \\
\hline Total & $\mathbf{4 4 3 . 8}$ \\
\hline
\end{tabular}

Source: Service statistique de la DDC, novembre 2002

Note: les contributions aux institutions financières internationales sont enregistrées au moment de l'émission des effets payables à vue. 


\section{$\square$ Coopération multilatérale avec des institutions de développement}

et d'aide humanitaire du système des Nations unies

Dans le domaine du développement, les principaux partenaires de la Suisse au sein du système des Nations unies demeurent le PNUD (52 millions de francs), l'UNICEF (17 millions), le HCR (13 millions) et le FNUAP (12 millions). La Suisse est actuellement membre du conseil d'administration du PNUD/FNUAP et de celui de l'UNICEF. En 2002, elle a assumé la vice-présidence du Conseil de l'UNICEF et y a assuré la coordination des pays donateurs. Elle a continué à appuyer l'ONUSIDA, l'UNIFEM et les VNU ainsi que plusieurs programmes de recherche de l'OMS.

\section{$\square$ Groupe de la Banque mondiale, banques de développement régionales et FIDA}

\section{Groupe de la Banque mondiale}

Après plusieurs tentatives, les négociations relatives à la treizième reconstitution du capital de l'Association internationale de développement (AID), filiale de la Banque mondiale, ont finalement abouti. La Banque mondiale pourra désormais verser environ $20 \%$ de ses fonds sous la forme de dons (au lieu de prêts à taux préférentiels). La Suisse, qui participera à cette reconstitution à hauteur de 2,43\% ou 530 millions de francs, a fêté cette année - en présence de J. Wolfensohn, président de la Banque mondiale - le $10^{\mathrm{e}}$ anniversaire de son adhésion aux Institutions de Bretton Woods. Pietro Veglio (DDC) a pris la succession de Matthias Meyer (seco) au conseil d'administration de la Banque mondiale. Pour 2001, la Suisse a déposé 140 millions de francs à la BNS à titre d'obligation envers la Banque mondiale.

DD Annuaire 2003, n ${ }^{\circ}$ 1, sous-chap. 7.4.2 «Banque mondiale».

\section{Banques de développement régionales et FIDA}

La Suisse a versé en 2001 un montant total de 73 millions de francs sous forme d'obligations aux banques de développement régionales, dont 46 millions au Fonds de la Banque africaine de développement et 13,9 millions au Fonds de la Banque asiatique de développement, ainsi que 17 millions au FIDA.

Le Conseil fédéral a nommé en mars 2002 Serge Chapatte, sous-directeur de la DDC, en qualité de représentant de la Suisse comme gouverneur au Fonds international de développement agricole (FIDA) ainsi que comme vice-gouverneur à la Banque africaine de développement (BAfD); et Adrian Schläpfer (DDC), Peter Bischof (DDC) et Martin Rohner (seco) en tant que vice-gouverneurs à la Banque asiatique de développement (BAsD), à la Banque interaméricaine de développement (BID) et au FIDA. Le gouverneur suisse pour ces trois banques reste l'ambassadeur Oscar Knapp, du seco.

La Suisse dispose auprès de la Banque africaine de développement de 1,467\% des voix et fait partie d'un groupe de vote comprenant également les quatre Etats scandinaves et l'Inde. Elle y est représentée depuis août 2002 par Thomas Eggenberger (seco) en qualité d'administrateur suppléant. Le Conseil fédéral a approuvé en juin 2002 la participation de la Suisse à une mobilisation intérimaire de ressources dans le cadre de la $9^{\mathrm{e}}$ reconstitution du Fonds africain, par une contribution de 44 millions de francs. Les négociations relatives à cette $9^{e}$ reconstitution n'ont pu être finalisées comme prévu pour la fin 
2001, en raison de sérieuses divergences entre les Etats-Unis et les pays donateurs européens sur la part de dons que le Fonds est censé octroyer ${ }^{36}$.

Banque asiatique de développement: la Suisse y détient $0,814 \%$ des voix et fait partie d'un groupe de vote incluant Belgique, France, Italie, Espagne et Portugal. Tadao Chino, président de la BAsD, a rendu visite à la Suisse en février 2002.

Banque interaméricaine de développement: la Suisse forme un groupe de vote avec la Belgique, l'Allemagne, Isräl, l'Italie et les Pays-Bas, et sa part de voix se chiffre à $0,47 \%$; elle met actuellement à disposition le conseiller (Robert Pantzer, seco) de l'administrateur représentant ce groupe de vote. Le président de la BID, Enrique V. Iglesias, a fait une visite à la Suisse en janvier 2002.

Banque européenne pour la reconstruction et le développement : la Suisse détient $2,28 \%$ du capital social de cette institution dont le siège est à Londres et elle représente un groupe de vote incluant le Liechtenstein, la Turquie, la République fédérale de Yougoslavie, l'Azerbaïdjan, le Kirghizistan, le Turkménistan et l'Ouzbékistan (actuellement dirigé par Laurent Guye, seco). Jean Lemierre, président de la BERD, a rendu visite à la Suisse en octobre 2002. Lemierre et le conseiller fédéral Couchepin ont signé à cette occasion un accord relatif à une contribution suisse de 2 millions de francs destinée au Programme de facilitation des échanges (Trade Facilitation Programme) de la BERD en Ouzbékistan, lequel fait partie d'un programme de promotion du commerce en Asie centrale ${ }^{37}$.

\section{$\square$ Coopération avec d'autres institutions multilatérales}

La Suisse compte toujours parmi les dix principaux pays donateurs aux centres de recherche agricole appartenant au Groupe consultatif pour la recherche agricole internationale (CGIAR); elle leur a versé en 2001 des contributions générales totalisant 11,1 millions de francs.

La Suisse, pays cofondateur du Fonds pour l'environnement mondial (FEM), a participé aux négociations qui ont abouti en octobre 2002 au réapprovisionnement du Fonds pour les quatre années à venir; elle a annoncé une contribution de 99 millions de francs (part égale à celle qu'elle verse à l'AID, soit 2,43\%). Sur ses versements de 2001, un total de 10,6 millions de francs relève de l'aide publique au développement.

Le nouveau Fonds global de lutte contre le sida, la tuberculose et le paludisme (Global Fund to Fight AIDS, Tuberculosis and Malaria, GF-ATM) a démarré à Genève en janvier 2002. Il se concentrera sur la mobilisation de fonds et sur le financement de programmes nationaux dans ces domaines. Les contributions promises atteignent actuellement 2 milliards de dollars. C'est aux pays bénéficiaires qu'incombe de réaliser les programmes ou projets financés par le GFATM. La Suisse s'est engagée à verser une première contribution de 10 millions de dollars; elle forme avec la Grande-Bretagne et le Canada un groupe de vote au sein du Comité directeur du GF-ATM. Un premier versement de 3 millions de dollars a été effectué fin 2001.

La Suisse continue par ailleurs de participer activement aux travaux de l'Agence internationale de la francophonie, qu'elle a soutenus en 2001 par des contributions générales totalisant 4,4 millions de francs.

36 seco, Participation de la Suisse à une mobilisation intérimaire de ressources dans le cadre de la $9^{e}$ reconstitution du Fonds africain de développement, communiqué de presse, du 26.6.02,

37 Conseil fédéral, Visite du Président de la BERD en Suisse, communiqué de presse, du 25.10.02. 


\subsection{AIDE DES ONG SUISSES}

La Suisse possède un tissu associatif très dense dans le domaine de la coopération et l'aide au développement, ou des relations Suisse-Sud plus généralement. En effet, on dénombre environ 600 ONG de développement ou d'aide d'urgence, associations de commerce équitable, groupements de solidarité, etc., dont un peu plus de 250 conduisent des projets dans les pays en développement ou de l'Est ${ }^{38}$. En 2001, les ONG suisses ont versé environ 523 millions de francs d'aide au développement dans les pays du Sud ainsi que dans les pays de l'Est. Les ONG ont récolté auprès du public 333 millions de francs, soit environ $64 \%$ des fonds qu'elles gèrent, et ont bénéficié des contributions publiques (Confédération, cantons et communes) pour un montant de 190 millions de francs (36\%).

\section{$\square$ Principales $O N G$ suisses actives dans le domaine de l'aide}

Environ 250 ONG suisses de développement conduisent des projets dans les pays du Sud et de l'Est. Le tableau ci-dessous présente les 12 ONG dont les dépenses effectives effectuées en 2001 sont supérieures à 10 millions de francs suisses. A noter également que certaines ONG sont beaucoup plus dépendantes de subventions que d'autres, ainsi Helvetas, Swisscontact ou encore l'Institut tropical suisse sont-ils subventionnés à hauteur de plus ou moins $90 \%$, alors que d'autres (Comité suisse pour l'UNICEF, World Vision Suisse) ne bénéficient d'aucune contribution publique. Ces situations très différentes s'expliquent par les relations historiques qu'entretient la Confédération avec certaines ONG ou par le fait que certaines ONG conduisent des projets en régie pour la Confédération.

Tableau 9: Versements d'aide des ONG en 2001 (en millions de francs)

\begin{tabular}{lcccc}
\hline & Total & Fonds propres & Contributions & $\begin{array}{r}\text { \% des contributions/ } \\
\text { versement d'aide }\end{array}$ \\
\hline Caritas & 51.4 & 40.0 & 11.4 & 22.2 \\
\hline Helvetas & 43.2 & 5.8 & 37.4 & 86.6 \\
\hline Entraide protestante (EPER) & 32.4 & 28.9 & 3.6 & 11.1 \\
\hline Swisscontact & 28.6 & 2.7 & 25.9 & 90.6 \\
\hline Croix-Rouge suisse & 26.5 & 17.6 & 8.9 & 33.6 \\
\hline Médecins sans frontières Suisse & 26.1 & 22.7 & 3.3 & 12.6 \\
\hline Fondation Terre des Hommes & 22.8 & 17.5 & 5.3 & 23.2 \\
\hline Comité suisse pour I'UNICEF & 21.5 & 21.5 & 0.0 & 0.0 \\
\hline Fondation pour I'Hôpital & 19.2 & 16.2 & 3.0 & 15.6 \\
des enfants de Kantha Bopha & 17.5 & 0.7 & 16.9 & 96.6 \\
\hline Institut tropical suisse & 17.4 & 4.2 & 13.2 & 0.0 \\
\hline MEDAIR & 12.6 & 12.6 & 0.0 & 75.9 \\
\hline World Vision Suisse & & & & 0.0 \\
\hline
\end{tabular}

Source: DDC, Aide suisse aux pays en développement et aux pays en transition 2001 (à paraître).

\section{$\square$ Principaux pays d'intervention des ONG suisses}

Les ONG suisses ont soutenu des projets dans plus de 142 pays en 2001, principalement dans les pays du Sud et dans les pays de l'Est considérés en développement (322,2 millions de francs, soit 96,7\%). Le solde des versements (11,3 millions de francs, soit 3,3\%) est destiné en grande majorité aux pays de l'Est en

38 Selon la base de données de l'Institut universitaire d'études du développement. 
transition (entre autres Russie, Roumanie, Ukraine et Bulgarie) et, dans une infime mesure, à certains pays plus avancés (Israël, Hongkong, Taïwan, Chypre).

Tableau 10: Principaux pays d'intervention des ONG suisses en 2001 (millions de francs)

\begin{tabular}{lccc}
\hline Pays de destination & Total & Fonds propres & Contributions \\
\hline Serbie & 35.2 & 27.2 & 8.0 \\
\hline Inde & 24.2 & 20.7 & 3.5 \\
\hline Cambodge & 24.4 & 20.0 & 4.4 \\
\hline Mozambique & 18.9 & 10.8 & 8.1 \\
\hline Soudan & 17.4 & 10.7 & 6.8 \\
\hline Congo, Rép. démocratique & 11.7 & 4.5 & 7.3 \\
\hline Brésil & 10.0 & 8.5 & 1.5 \\
\hline Bolivie & 9.5 & 5.6 & 4.0 \\
\hline Népal & 9.5 & 1.4 & 8.1 \\
\hline Vietnam & 9.4 & 3.8 & 5.6 \\
\hline Pérou & 9.3 & 5.4 & 3.9
\end{tabular}

Source: DDC, Aide suisse aux pays en développement et aux pays en transition 2001 (à paraître).

\section{$\square$ Principales campagnes de sensibilisation des ONG suisses} aux politiques de développement (2002)

Dans un autre registre, les ONG constituent un groupe de pression incontournable, tout en menant des campagnes de sensibilisation auprès du public suisse. En 2001, la Communauté de travail des œuvres d'entraide ${ }^{39}$ a mené deux campagnes importantes en vue de votation populaire. La première, en mars 2002, en faveur de l'adhésion de la Suisse à l'Organisation des Nations unies, adhésion acceptée par le peuple suisse à une courte majorité de 54,5\%. La seconde campagne concernait la Fondation Suisse solidaire (voir ci-dessous), largement soutenue par les œuvres d'entraide. Les résultats de la votation de septembre 2002 furent sans appel; le peuple suisse a clairement rejeté, à une double majorité du peuple et des cantons, cette fondation.

En 2001, la Déclaration de Berne ${ }^{40}$ a poursuivi sa campagne contre la privatisation des services publics, de plus elle a lancé une action contre l'utilisation de pesticides dangereux dans les pays du Sud «Halte au Paraquat».

La campagne «Clean Clothes ${ }^{41}$, pour des habits produits dans la dignité, menée par Action de Carême, la Déclaration de Berne et Pain pour le prochain, s'est poursuivie en 2001 par la mise en place d'un projet pilote dont le but est de tester un modèle de vérification indépendante de codes de conduite.

\subsection{FONDATION SUISSE SOLIDAIRE}

En septembre 2002, le peuple et les cantons ont clairement refusé la création de la Fondation Suisse solidaire. Cette fondation, proposée par le gouvernement, avait pour objectif de soutenir des projets permettant de lutter contre la pauvreté et la violence, tant en Suisse qu'à l'étranger. Elle devait être financée par une partie des bénéfices engendrés par la vente de l'or excédentaire de la Banque

39 Site de la Communauté de travail des œuvres d'entraide : <www.swisscoalition.ch $>$.

40 Site de la Déclaration de Berne: <www.evb.ch $>$.

41 Site de la campagne: <www.cleanclothes.ch>. 
nationale suisse. Le texte du Conseil fédéral prévoyait de répartir le produit de la vente des 1300 tonnes d'or excédentaires à parts égales entre la Fondation, l'AVS et les cantons. $51,8 \%$ des votants et 20 cantons ont rejeté le texte du Conseil fédéral, contre-projet à une initiative de l'UDC. L'Union démocratique du centre avait lancé une initiative qui visait à verser l'ensemble du produit de la vente de l'or au système public de sécurité sociale, l'AVS, initiative également balayée par $52,4 \%$ des votants et 17 cantons $^{42}$. La déception a été très vive parmi le collectif des œuvres d'entraide et des mouvements de jeunesse qui avaient mené une active campagne en faveur de la Fondation Suisse solidaire.

ASTM 2002, Projet de Fondation Suisse solidaire, pp. 240-241.

\subsection{PÔLE DE RECHERCHE NATIONAL NORD-SUD}

Le Conseil fédéral et le Parlement ont introduit en 1999, à la demande du Fonds national suisse de la recherche scientifique (FNS), un nouveau mécanisme de soutien pour la recherche scientifique, qui a mené à la création de divers pôles de recherche nationaux (NCCR: National Centers of Competence in Research), d'une durée maximale de douze ans.

Le NCCR North-South est l'un des 14 pôles de recherche nationaux mis en place par le FNS. Lancé en 2001, ses activités regroupent 250 chercheurs de Suisse, d'Amérique latine, d'Afrique et d'Asie. Sept institutions suisses ${ }^{43}$ portent la responsabilité du projet; le Centre pour l'environnement et le développement de l'Université de Berne en assure la direction ${ }^{44}$. L'Institut universitaire d'études du développement à Genève est responsable du volet sur la gouvernance ${ }^{45}$.

Comme le souligne la brochure de présentation du projet, «la philosophie du NCCR North-South accorde une grande importance à l'équilibre entre le Nord et le Sud ${ }^{46}$. Chaque institution suisse impliquée collabore, en moyenne, avec trois ou quatre partenaires dans le Sud. Les partenaires suisses sont financés par le FNS, alors que la DDC apporte un financement égal aux partenaires des pays en voie de développement et de transition. Les chercheurs du Nord et du Sud définissent ensemble les projets de recherche. Alors que le cadre conceptuel a été établi au Nord, un poids plus grand est mis sur la formation des scientifiques dans le Sud.

Le NCCR North-South a pour titre «Partenariats de recherche pour atténuer les syndromes du changement global». Selon l'un des responsables du projet,

42 «Or de la BNS : pas de solidarité avec le Conseil fédéral», Le Temps, 23.9.02.

43 Centre pour le développement et l'environnement (CDE), Université de Berne; Development Study Group, Institut de géographie, Université de Zurich; Institut tropical suisse (ITS), Bâle; Institut universitaire d'études du développement (iuéd), Genève; Laboratoire de sociologie urbaine (LASUR), EFFL Lausanne; Département eau et assainissement dans les pays en développement (SANDEC), Dübendorf; Fondation Suisse pour la paix (Swisspeace), Berne.

44 La coordination opérationnelle du programme est conduite par le Management Center NCCR NorthSouth, CDE-GIUB, Steigerhubelstrasse 3, 3008 Berne. Tél. 031.631.52.72. Pour de plus amples informations sur le NCCR North-South: <www.nccr-north-south.unibe.ch>.

45 Pour de plus amples informations sur le projet «Gouvernance, développement humain et environnement » : <www.nccr-ip8.org >.

46 NCCR North-South, Partenarias de recherche pour atténuer les syndromes du changement global, Pôle de recherche national Nord-Sud, Berne: DDC et FNS, 2002. 
«l'approche par syndrome se base sur l'hypothèse que des ensembles similaires de problèmes clés surgissent partout dans le monde et que des approches similaires pour les atténuer peuvent être trouvées ${ }^{47}$. Les études de terrain s'effectuent dans huit régions d'Asie, d'Amérique latine et d'Afrique, et les projets spécifiques (Individual Projects = IP) portent, outre le cadre conceptuel et la méthodologie (IP1), sur les ressources naturelles et l'écologie (IP2); l'eau, l'assainissement environnemental et l'agriculture urbaine (IP3); la santé et le bienêtre (IP4); les pratiques sociales et l'empowerment dans les sociétés urbaines (IP5); les changements institutionnels et les stratégies de subsistance (IP6); les changements environnementaux et la transformation de la «culture du conflit» (IP7); la gouvernance, le développement humain et l'environnement (IP8).

\section{SOURCES}

\section{Publications d'organisations internationales}

$\mathrm{CAD}$, Examen en matière de coopération pour le développement, Suisse, Les Dossiers du CAD - Coopération pour le développement, CAD 2000, vol.1, n 4, Paris: OCDE, 2001.

OCDE, Les Dossiers du CAD - Coopération pour le développement - Rapport 2001, vol. 3, $\mathrm{n}^{\circ} 1$, Paris: OCDE, 2002.

OCDE, Les lignes directrices du CAD. Intégrer les conventions de Rio pour la coopération au développement, Paris: OCDE, 2002.

OCDE, Les lignes directrices du CAD. La réduction de la pauvreté, Paris: OCDE, 2001.

$\mathrm{OCDE}$, Les lignes directrices du CAD. Prévenir les conflits violents: quels moyens d'action?, Paris: OCDE, 2001.

OCDE, Les lignes directrices du CAD. Renforcer les capacités commerciales au service du développement, Paris: OCDE, 2001.

OCDE, Les lignes directrices du CAD. Stratégies de développement durable, Paris: OCDE, 2001.

OCDE, communiqués de presse parus en 2002.

\section{Publications officielles suisses}

Conseil fédéral, Lignes directrices Nord-Sud. Rapport sur les relations Nord-Sud de la Suisse dans les années 90 du 7 mars 1994.

Conseil fédéral, Message concernant la continuation du financement et la réorientation des mesures de politique économique et commerciale au titre de la coopération au développement du 29 mai 1996.

DDC, Les Montagnes et leurs habitants, Berne: DDC, 2002.

DDC, Newsletter, publiées en 2002.

DDC, Un seul monde, publiés en 2002.

DDC, communiqués de presse parus en 2002.

DDC/seco, Coup d'œil aux Cadres stratégiques de lutte contre la pauvreté, 2002.

DDC/seco, Mémorandum de la Suisse au CAD, Berne, avril 2000.

DDC/seco, Rapport annuel 2001 - Coopération internationale de la Suisse, Berne, 2002.

NCCR North-South, Partenariats de recherche pour atténuer les syndromes du changement global. Pôle de recherche national Nord-Sud, DDC et FNS, Berne, 2002.

seco, Domaine de prestation Développement et transition - Stratégie 2006, Berne, mars 2002.

La Vie économique, «Nouvelle stratégie du seco dans le domaine du développement et de la transition», mai 2002.

\section{SITES INTERNET}

Comité d'aide au développement de l'OCDE: <www.oecd.org/dac>.

Direction du développement et de la coopération (DDC): <www.deza.admin.ch $>$.

Secrétariat d'Etat à l'économie (seco) : <www.seco.admin.ch>. 